\title{
Versatile Macroscale Concentration Gradients of Nanoparticles in Soft Nanocomposites
}

\author{
Patricia Taladriz-Blanco, Barbara Rothen-Rutishauser, Alke Petri-Fink, and Sandor Balog*
}

Nanocomposite materials benefit from the diverse physicochemical properties featured by nanoparticles, and the presence of nanoparticle concentration gradients can lend functions to macroscopic materials beyond the realm of classical nanocomposites. It is shown here that linearity and time-shift invariance obtained via the synergism of two independent physical phenomena-translational self-diffusion and shear-driven dispersionmay give access to an exceptionally high degree of flexibility in the design of scalable and programmable long-range concentration gradients of nanoparticles in solidifiable liquid matrices.
The history of functionally graded materials began far from the realm of soft matter, ${ }^{[1-3]}$ yet the emerging promise of biomimicry, biomimetics, and eventually true bioinspiration, ${ }^{[4,5]}$ brought the necessity to design and fabricate smooth macroscale-gradients also in polymer- supramolecular-, and soft nanocomposites. ${ }^{[6,7]}$ Conventional nanocomposites merge the specific optical, magnetic, thermal, catalytic and mechanical properties of nanoparticles (NPs) with the properties of the surrounding matrix, resulting in macroscopically homogenous hybrid materials. ${ }^{[8,9]}$ Spatial NP gradient profiles, such as concentration, size, shape, and orientation can add additional degrees of freedom to soft condensed matter designs, and thus, they represent a considerable interest. ${ }^{[10]}$

Recently, beside translational self-diffusion ${ }^{[11]}$ and buoyancydriven gradient creation, ${ }^{[12]}$ analytical ultracentrifugation was presented as one of the most versatile approaches to design and create macroscale concentration gradient profiles in liquid matrices that were solidified subsequently by gelation. ${ }^{[13]}$ Depending on the relative rate of sedimentation and diffusion, different concentration gradients are generated: for example, sigmoidal profiles were obtained at high centrifugal forces where sedimentation governed the concentration profile, and

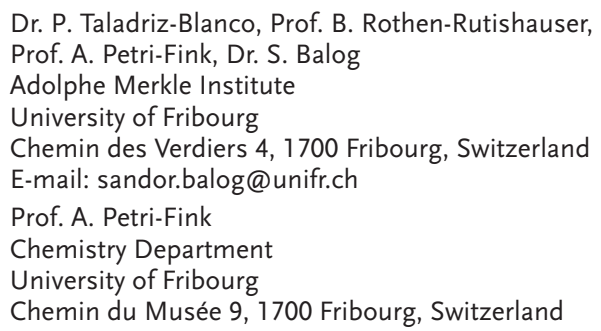

The ORCID identification number(s) for the author(s) of this article can be found under https://doi.org/10.1002/smll.201905192.

DOI: 10.1002/smll.201905192 exponential concentration gradients were generated when sedimentation and diffusion were in equilibrium at lower centrifugal forces. ${ }^{[13]}$

This is a big step in the design of soft nanocomposites for it enables that such monotonic gradient profiles can be modeled quantitatively and designed precisely before processing-owing to the availability of a mathematical framework that is able to model faithfully the related phenomena. Following this concept, i.e., that 1) the creation and fixation of macroscale gradient profiles may be decoupled and 2) may be designed and tailored accurately by mathematical means, we present an approach dedicated to creating continuous and smooth long-range longitudinal concentration gradients of NPs in macroscopic 1D soft materials, such as fibers. The available design-space is rich, and includes either symmetric or asymmetric, monotonic or nonmonotonic, linear, or nonlinear profiles, with the possibility of, e.g., periodicity, having multiple local minima and maxima with either complementary or orthogonal functionalities.

The physical phenomenon our approach is based on is called Taylor dispersion, which can be accurately described by the asymptotic solution of the convective-diffusion equation ${ }^{[14-16]}$ corresponding to the geometry of the flow channels. ${ }^{[17]}$ Taylor dispersion refers to the enhanced dispersion of particles in a steady Poiseuille flow, where the linear shear stress creates a quadratic flow-velocity profile, and induces a spontaneous net transport of particles via translational self-diffusion. The rate and overall extent of spread is a function of the unidirectional velocity profile and translational self-diffusion coefficient of the particles. Taylor dispersion is characteristic to laminar flows and scalable on a wide range of dimensions. In the case of NPs, the phenomenon can be easily realized in a microcapillary laminar flow, which also provides an outstanding experimental technique to characterize the hydrodynamic radius of organic and inorganic NPs. ${ }^{[18-26]}$

Although from the theoretical point of view, its dimensionality is scalable, Taylor dispersion is essentially a microfluidic technique, and microfluidics offer an excellent platform to create a gradient, e.g., via electrospinning. ${ }^{[27]}$ Indeed, Taylor dispersion was connected to the creation of simple concentration gradients more than ten years ago, ${ }^{[28-30]}$ yet it was shown only recently that Taylor dispersion is de facto a casual linear timeinvariant system. ${ }^{[31]}$ We show here that this mathematical property has important implications for the applicability of Taylor dispersion when creating macroscale gradient profiles of NPs, because the gradient profile of NPs can be designed accurately 


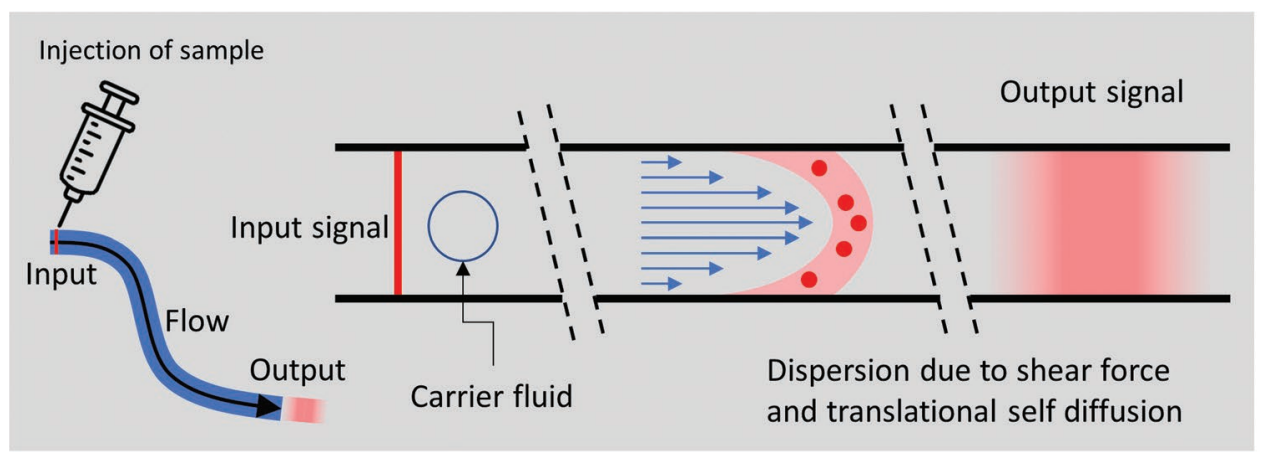

Figure 1. Taylor dispersion as a signal system. The injected narrow band of a dispersion of NPs is dispersed by the interplay of the pressure-driven laminar flow of the carrier fluid and the translational self-diffusion of the particles, while traveling the distance between injection (input) and detection point (output). Taylor dispersion is a causal process: the output depends only on the current and past input, but not on future inputs. Taylor dispersion is also time-invariant: a given time-delay on the input results in the same time-delay on the output. Furthermore, Taylor dispersion is linear: changing the input in a linear way will change the output in the same linear way. Accordingly, any linear combination of distinct inputs will produce the same linear combination of the related outputs.

in advance, and the design-space is vast: it enables arbitrary profiles and virtually unlimited flexibility.

First, we discuss the mathematical background of Taylor dispersion as a signal system (Figure 1). Briefly, if the system is 1) casual, 2) linear, and 3) time invariant, the temporal output signal is the temporal input signal convolved with the impulse response function. For the sake of simplicity, our discussion will be restricted to circular flow channels, which, however, does not represent either a necessity or a boundary of applicability. We define the output signal as the NP concentration averaged over the cross section of the channel. Then, the impulse response function of Taylor dispersion of uniform particles of hydrodynamic radius $r$ may be very well approximated by a Gaussian function ${ }^{[14,15]}$

$h(t)=\frac{1}{\sqrt{2 \pi} \sigma} \mathrm{e}^{-\frac{\left(t-t_{0}\right)^{2}}{2 \sigma^{2}}}$

when $t_{0} / \sigma>10$ and $\sigma=\sqrt{\frac{\pi \eta Y^{2}}{4 k_{\mathrm{B}} T} \cdot r \cdot t_{0}}, Y$ the capillary radius, $\eta$ the viscosity of the fluid, $T$ the temperature, $k_{\mathrm{B}}$ the Boltzmann constant, and $t_{0}=l / v$ the so-called residence time defined by the distance between detection and injection points $(l)$ and the mean velocity of the flow $(v)$.

Taylor dispersion is a casual, linear, and time-invariant system. Accordingly, when the input is a given function of time $x(t)$, the output as a function of time $\gamma(t)$ can be accurately calculated as

$Y(t)=\int_{0}^{t} x(\tau) \cdot h(t-\tau) \mathrm{d} \tau$

The mathematical operation defined by Equation (2) is called convolution, and means that when one administers an either continuous or discontinuous succession of impulses of different amplitudes defined by the value of $x$, then $y$ is the output response to $x$. Accordingly, this relationship also means that the impulse response function $h(t)$ describes completely the relationship between the input and output signals $\gamma(t)$, and by programming $x(t)$, one can design and tailor $y(t)$, which is the concentration gradient profile of the NPs. In fact, one can even reverse the order, i.e., for creating a given output $\gamma(t)$, one can find the required input $x(t)$ via deconvolution, by using the convolution theorem. This mathematical property of Taylor dispersion offers yet-untapped opportunities in the design and creation of particle concentration gradients in quasi 1- and 2-D liquid matrices, such as in flat flow channels.

To illustrate the versatility, we design some output profiles using simple inputs profiles (Figure 2). If the input profile is that of a uniform distribution

$x(t)=\frac{1}{t_{2}-t_{1}}$

i.e., the concentration of the injection is constant, the outputas obtained via Equation (2) - is

$y(t)=\frac{\operatorname{Erf}\left[\frac{t_{1}+t_{0}-t}{\sqrt{2} \sigma}\right]-\operatorname{Erf}\left[\frac{t_{2}+t_{0}-t}{\sqrt{2} \sigma}\right]}{2\left(t_{2}-t_{1}\right)}$

When the input profile increases/decreases linearly from a concentration of $c_{1}$ to $c_{2}$ between $t_{1}$ and $t_{2}$ time, the input function is

$x(t)=\frac{c_{2} t_{1}-c_{1} t_{2}}{t_{1}-t_{2}}+\frac{\left(c_{1}-c_{2}\right)}{t_{1}-t_{2}} t$

where $c_{1} \geq 0$ and $c_{2} \geq 0$ (i.e., concentration cannot be negative), and the corresponding output particle concentration profile is

$Y(t)=\frac{\left(\operatorname{Erf}\left[\frac{t-\mu-t_{1}}{\sqrt{2} \sigma}\right]-\operatorname{Erf}\left[\frac{t-\mu-t_{2}}{\sqrt{2} \sigma}\right]\right)\left(c_{2}\left(\mu+t_{1}-t\right)+c_{1}\left(t-\mu-t_{2}\right)\right)}{2\left(t_{1}-t_{2}\right)}-\frac{1}{\sqrt{2 \pi}\left(t_{1}-t_{2}\right)}\left(\mathrm{e}^{-\frac{t^{2}+2 \mu t_{1}+t_{1}^{2}+\left(\mu+t_{2}\right)^{2}}{2 \sigma^{2}}}\left(\mathrm{e}^{\frac{2 \mu t_{1}+t_{1}^{2}+2 t\left(\mu+t_{2}\right)}{2 \sigma^{2}}}-\mathrm{e}^{\frac{2 t\left(\mu+t_{1}\right)+t_{2}\left(2 \mu+t_{2}\right)}{2 \sigma^{2}}}\right) \sigma\left(c_{1}-c_{2}\right)\right)$ 

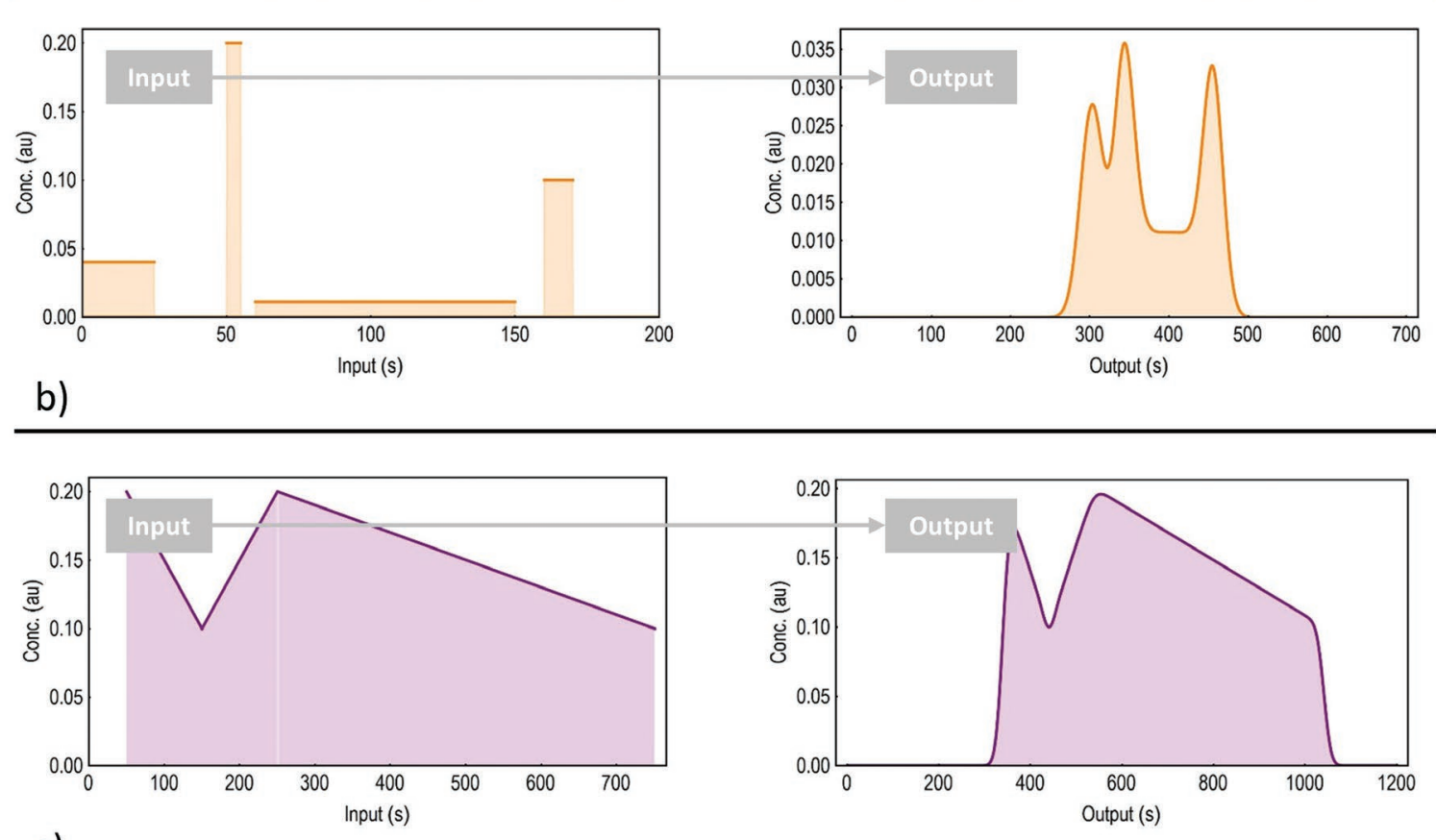

c)
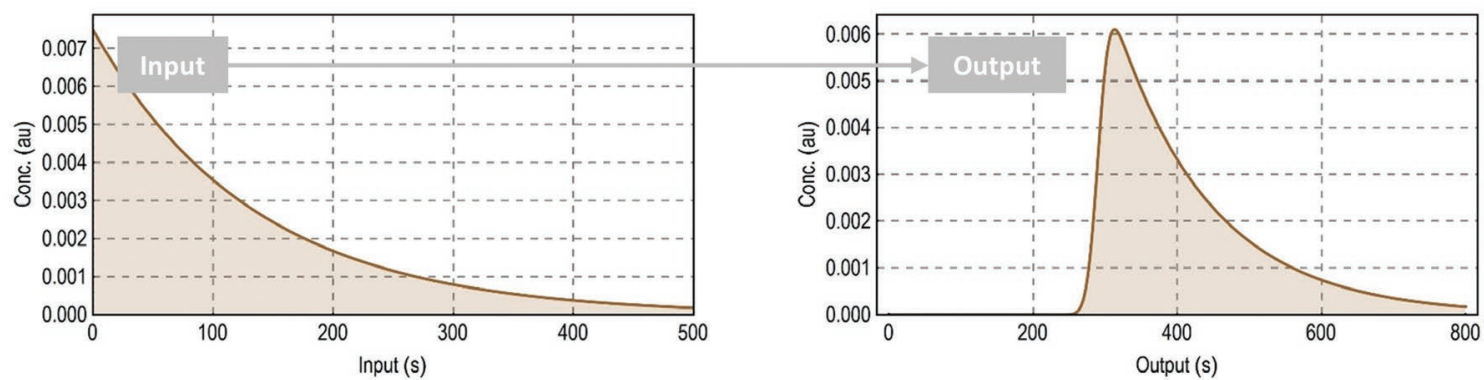

d)

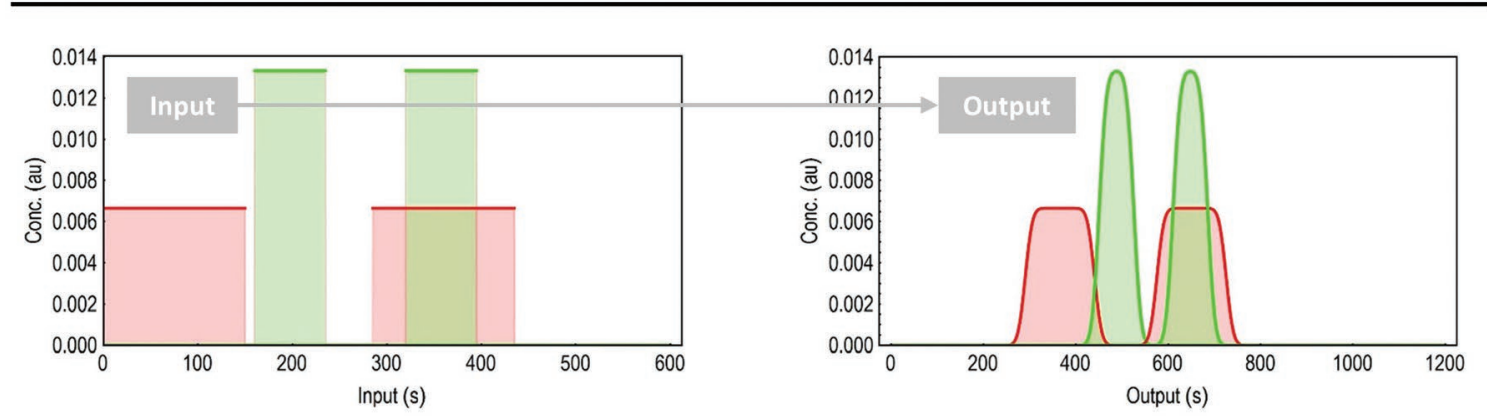

Figure 2. Input-output pairs of particle concentration profiles. a) Equations (3) and (4): the concentration of the input (injection) is constant b) Equations (5) and (6): the concentration of the input profile increases/decreases linearly, c) Equations (7) and (8): the concentration of the input profile decreases exponentially, and d) different particle system can be co-dispersed as wished, which enables the combination of complementary and orthogonal properties.

Finally, when the concentration of the input profile decreases the output particle concentration profile is exponentially

$x(t)=\lambda \mathrm{e}^{-\lambda t}$

(7) $\quad y(t)=\frac{\lambda}{2} \mathrm{e}^{\frac{\lambda}{2}\left(2 t_{0}+\lambda \sigma^{2}-2 t\right)} \operatorname{Erfc}\left[\frac{t_{0}+\lambda \sigma^{2}-t}{\sqrt{2} \sigma}\right]$ 
To draw these designs, the parameters $t_{0}$ and $\sigma$ were chosen to be fully realistic so that they correspond to aqueous colloidal systems: We modeled particles with a hydrodynamic radius of $5 \mathrm{~nm}$, in a cylindrical capillary with a total length of $L=$ $100 \mathrm{~cm}$ with a radius of $Y=70 \mu \mathrm{m}$, in water at room temperature $\left(25^{\circ} \mathrm{C}\right)$, with a pressure of $P=100$ mbar. According to these parameters, rearranging the Hagen-Poiseuille equation $v=Y^{2} P /(8 L \eta)$ determined a mean flow velocity of $1.72 \mathrm{~mm} \mathrm{~s}^{-1}$. We set the observation distance $l$ at $50 \mathrm{~cm}$, and thus, according to Equation 1, the residence time $t_{0}$ was $290.5 \mathrm{~s}$, and the of the width $\sigma$ of the impulse response function was $12.3 \mathrm{~s}$. We emphasize that these parameter choices were arbitrary, and not in any way represented a limit or restriction. The corresponding input-output pairs of particle concentration profiles are shown in Figure 2.

To illustrate the versatility, we also present an example of using the convolution theorem: Let $\phi$ denote the Fourier transform of a real-valued function, and $\phi^{-1}$ denote the inverse Fourier transform. Then, according to the convolution theorem: $x(t)=\phi^{-1}(\phi(\gamma) / \phi(h))$. For example, if the desired gradient profile (output) is a perfect sine function, being similar to a sinusoidal grating, $\gamma(t)=a+\sin (\omega t)$ where $a \geq 1$, the necessary input function must be $x(t, \omega)=\sqrt{2 \pi} a+\sqrt{2 \pi} \mathrm{e}^{\frac{\sigma^{2} \omega^{2}}{2}} \sin (\omega t+\omega \mu)$ as obtained via the convolution theorem. Now, given that sine functions can be used to design and create very specific and complex periodic profiles-which is usually referred to as Fourier synthesis-linear combinations of input signals, i.e., $\sum A_{i} x\left(t, \omega_{i}\right)$ where $A_{i}>0$, provide limitless combinations of sine functions $\sum A_{i} \gamma_{i}$, and hence, gives access to an exceptionally versatile particle-gradient design.

While both the fundamental principles and arbitrary special cases can be captured with exact mathematical models, to demonstrate experimentally the full versatility of the design of particle gradients is well beyond the capacity of our current Taylor dispersion setup. Our setup is dedicated to characterize accurately and precisely the hydrodynamic radius of small molecules, proteins, supramolecular complexes, macromolecules, nanoparticles, and their self-assembly. ${ }^{[22,25,31,32}$ Accordingly, our ability to modulate experimentally the temporal shape of the input signal (Figures 1 and 2 and $x(\tau)$ in Equation 2) is fairly limited at the present.

Nonetheless, to demonstrate experimentally the basic aspects, we studied experimentally a few cases. We used aqueous dispersions of 1) polymer-coated gold particles (PEGylated AuNPs with carboxylic acid (COOH) end group), 2) polymer-coated silver particles (PVP-coated AgNPs), and 3) their co-dispersion. In the first experiment, we created periodic gradients of AuNPs, in which the particle gradient profiles are modulated periodically (Figure 3 ).

Combining particle systems enables to impart the concentration gradients with either orthogonal or complementary properties, e.g., with magnetic and optical features. To show the linearity in gradient generation as well as the possibility of combining different particle systems, in the second experiments we created a single gradient peak where AuNPs and AgNPs were co-dispersed (Figure 4). We have performed five set of Taylor dispersion analyses: 1) Ag dispersion measured at $400 \mathrm{~nm}, 2) \mathrm{Au}$ dispersion measured at $400 \mathrm{~nm}, 3) \mathrm{Au}$ dispersion measured at $520 \mathrm{~nm}, 4) \mathrm{Au} / \mathrm{Ag}$ co-dispersion measured at
$400 \mathrm{~nm}$, and 5) $\mathrm{Au} / \mathrm{Ag}$ co-dispersion measured at $520 \mathrm{~nm}$. To prove the mutual presence of both AuNPs and AgNPs in the gradient profile, we relied on the fact that the AgNPs absorb strongly around $400 \mathrm{~nm}$, and weakly beyond $500 \mathrm{~nm}$, while the AuNPs absorb strongly around $520 \mathrm{~nm}$, owing to localized surface plasmon resonances. ${ }^{[33]}$ Therefore, the apparent particle size (absorption-weighted average) of the $\mathrm{Au} / \mathrm{Ag}$ co-dispersion was expected to be different when measured at these two wavelengths. When the measurement-Taylor dispersion analysis of nanoparticles ${ }^{[22]}$ — was performed at $400 \mathrm{~nm}$, the presence of AgNPs was clear, while when it was performed at $520 \mathrm{~nm}$, the presence of AuNPs was dominant.

To summarize, our study presents the challenging idea that Taylor dispersion-being a linear time-invariant system-can be used to create macroscale gradients of nanoparticles dispersed and co-dispersed in liquid matrices. In our theoretical approach, we dealt with inert and stationary particle systems, e.g., the shear-force and liquid matrix do not change either the hydrodynamic radius or the colloidal stability of the particle system. The idea we put forward addressed particles that are dispersed in a liquid matrix that is itself solidifiable, e.g., a lowviscosity UV-curable photopolymer with low curing shrinkage. Therefore, we did not consider the addition of any agent to impart this ability, although to prove the concept of creating gradients, we addressed aqueous systems, owing to the parameters of our Taylor dispersion setup.

Furthermore, we addressed particle systems where interparticle interactions are negligible, and accordingly, the simultaneous dispersion of different particle systems does not influence each other's output signals, in other words there is no interference between output signals. To achieve perfectly smooth and locally homogeneous long-range gradients, e.g. even spanning over a few meters is arguably difficult, ${ }^{[34]}$ and our study aims to change that. We showed that the related physical principles can be described by exact mathematical models, and consequently, particle gradients of exceptional versatility can be designed at wish. These scalable and programmable long-range concentration gradients can be fixed by solidifying the liquid matrix, e.g. either by gelation or resin curing or using one-component composites particles ${ }^{[35]}$ or stimuli responsive noncovalent supramolecular interactions. ${ }^{[36]}$

We believe that there is plenty of room for extending the versatility of such particle concentration gradients. For example, local hierarchical assemblies may be made available by using specific interactions between particles. ${ }^{[37,38]}$ Furthermore, the application of external fields, e.g., electromagnetic fields and anisotropic nanoparticles can further add to the versatility of the design via alignment, and local or global perturbations, to introduce, e.g., disorder and randomness. For the sake of simplicity, we discussed homogeneous inputs in this study, i.e., the input concentration was homogeneous across the cross section of the channel geometry. However, if the input can be modulated spatially-e.g., in the case of a cylindrical channels: radially (polar coordinates) -it attributes yet another degree of freedom to the versatility of the design. To achieve diverse modulated inputs, microfluidic techniques offer a vast toolbox for input generation, including, e.g., programmable sequences of composition ${ }^{[39]}$ and complex input waveforms, ${ }^{[40]}$ which enable co-dispersing different particles-even different carrier 

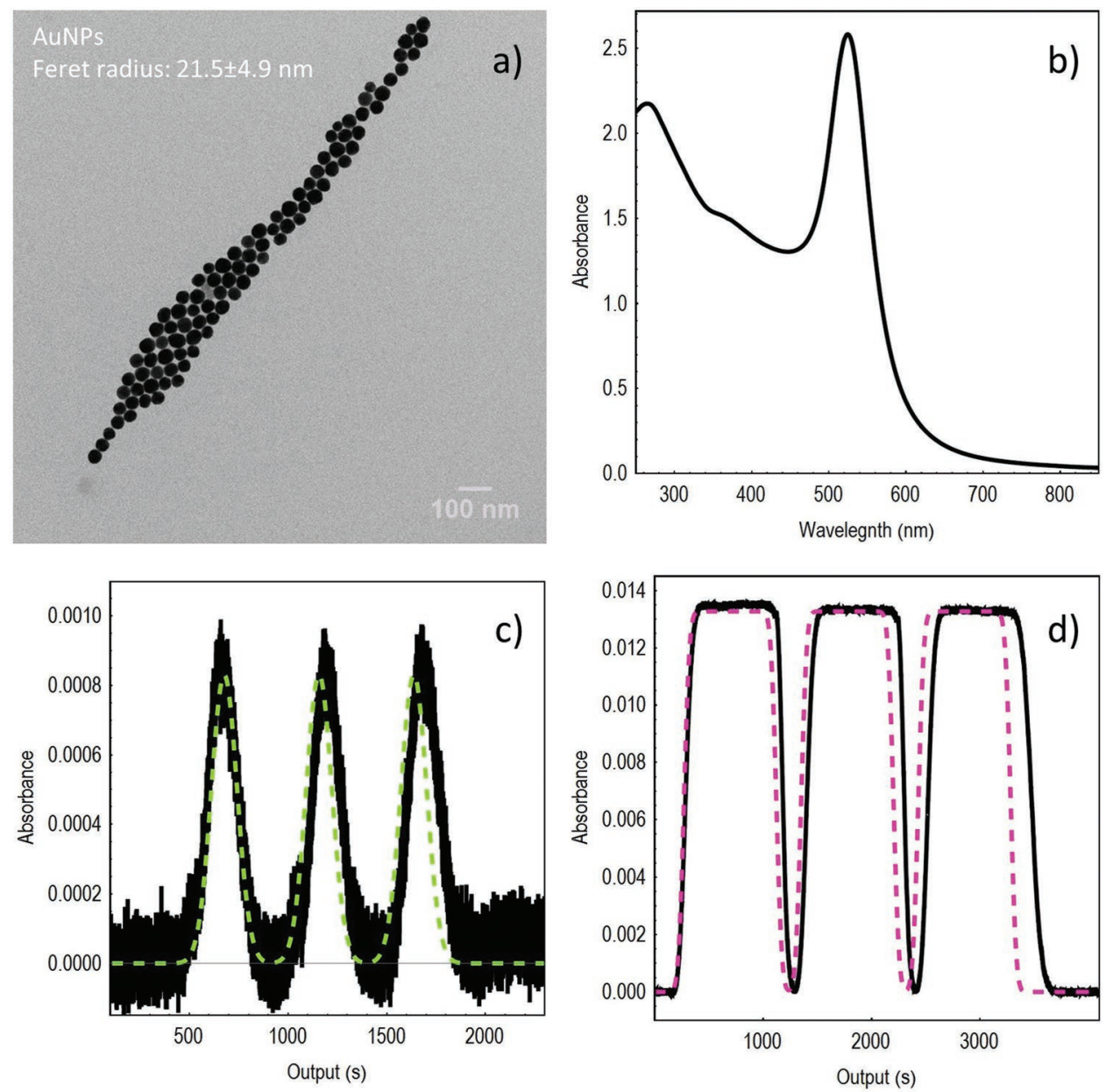

Figure 3. a) Transmission electron microscopy image and b) the UV-Vis spectrum of AuNPs with a mean core radius of $\approx 21$ nm (Feret radius). c,d) Output gradient profiles resulting from periodic inputs corresponding to Equations (3) and (4). In these experiments, alternating sequences of AuNPs and Milli-Q water were injected into the capillary as input. c) AuNPs 200 mbar for 0.08 min and then Milli-Q water 90 mbar for 8 min, repeated three times (input length was $5 \mathrm{~s}$ and consecutive input events were set apart by $8 \mathrm{~min}$ ). The dashed line indicates the theoretically expected profile. d) AuNPs 200 mbar for $10 \mathrm{~min}$ and then Milli-Q water $90 \mathrm{mbar}$ for $8 \mathrm{~min}$, repeated three times (input length was 10 min and consecutive input events were set apart by $8 \mathrm{~min}$.). The dashed line indicates the theoretically expected profile. We point out that at the moment we are unable to perform experiments with input profiles corresponding to Figure $2 b, c$, simply because we do not have the experimental means to create and control accurately such input profiles.

fluids-in a composition- time-, and position-shared manner. Furthermore, the velocity of a laminar flow in a tapered channel would systematically increase/decrease (narrowing/ widening channel radius), even if the driving pressure remains constant. Therefore, the interplay between time-dependent pressure and a nonconstant velocity could offer interesting possibilities for creating non-conventional concentration-gradients in such geometries.

Last but not least, as mentioned above, Taylor dispersion is scalable, which means that as long as i) the flow is laminar, ii) the residence time is much larger than the characteristic time required for the particles to diffuse across the capillary $\left(D t_{0} / Y^{2}<1.4\right)$ and iii) the transport rate due to axial diffusion is negligible compared to dispersion due to shear $(v Y / D<69)$, the theory outlined here is complete and valid. ${ }^{[14-16]}$ Until the Reynolds number $(R e)$ is below 2100 , the flow remains laminar. By using the Hagen-Poiseuille equation, one can express this condition by a straightforward inequality: $R e=P Y^{3} \rho$ / $\left(4 L \eta^{2}\right)<2100$, where $L$ is the total length of the flow-channel. Accordingly, the number of possible combinations to meet this condition is vast, and for a given channel-geometry (radius $Y$ and length $L$ ) and given fluid type (viscosity $\eta$ and mass density $\rho$ ) one can set the driving pressure accordingly. As an example, if we consider NPs with a hydrodynamic diameter of $10 \mathrm{~nm}$, dispersed in a long cylindrical channel with a radius of $0.5 \mathrm{~mm}$ and a length of $15 \mathrm{~m}$, in water at a temperature of $37^{\circ} \mathrm{C}$, driven by a pressure of 3 mbar, the smooth concentration gradient profile will span over more than $1 \mathrm{~m}$ in the middle of the channel.

Finally, while in our case the primary parameter was timeowing to the original mathematical formulation-, time as parameter can be converted into distance by using the flow velocity, and the transformation of the related mathematical expressions is straightforward. 

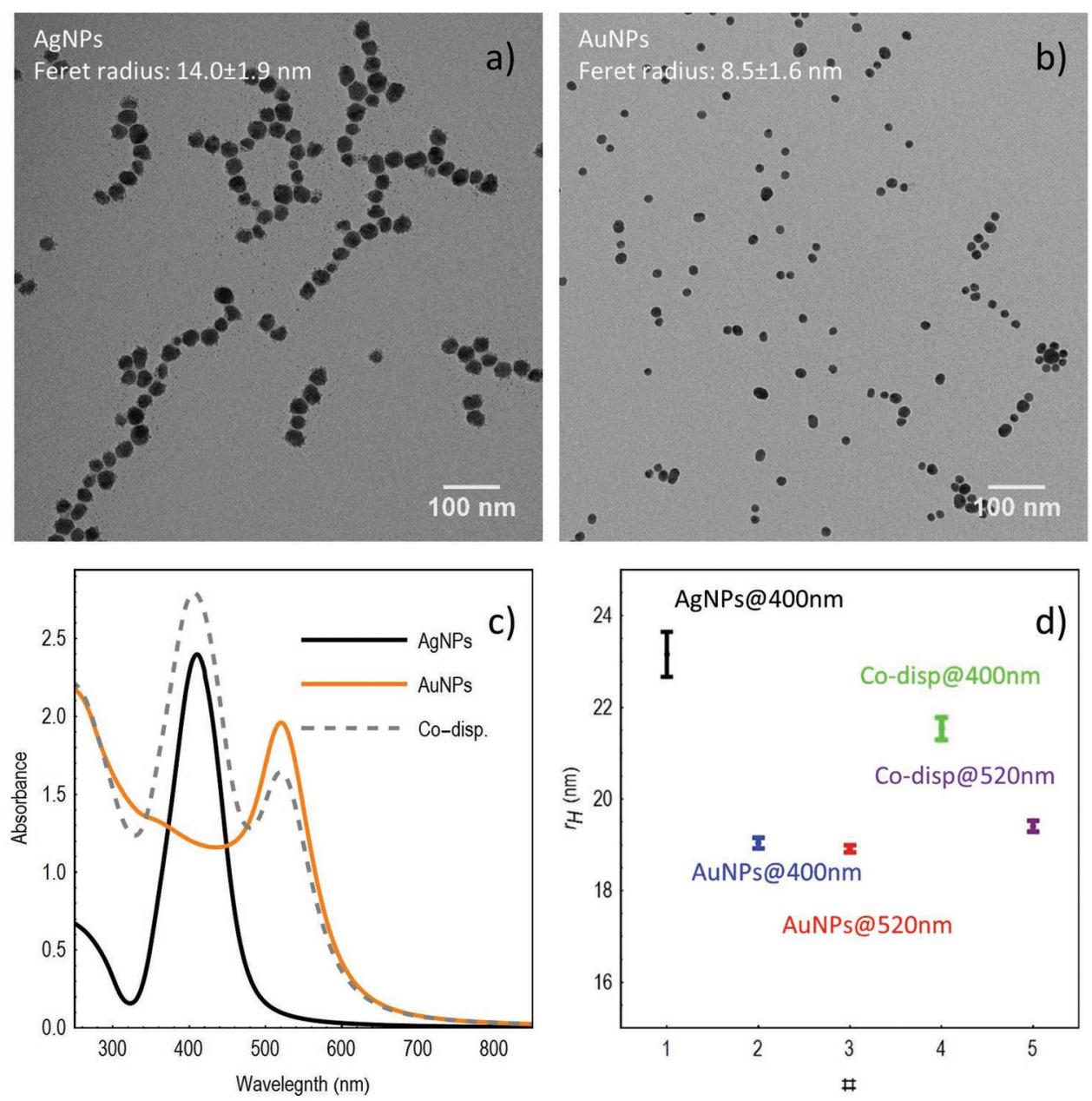

Figure 4. The analysis of single gradient profiles of co-dispersed AgNPs and AuNPs. a,b) Transmission electron microscopy images of the AgNPs and AuNPs. c) The UV-vis spectra of single and co-dispersed AgNPs and AuNPs ([Ag] $\left.=[\mathrm{Au}]=0.6 \times 10^{-3} \mathrm{M}\right)$. d) Taylor dispersion analysis of single and co-dispersed AgNPs and AuNPs. Data points (mean and standard deviation) are statistics of five measurements. In this experiment, five sets of Taylor dispersion analyses were performed: In the first one (\#1), the dispersion of the AgNPs was measured at $400 \mathrm{~nm}$, in the second one (\#2), the dispersion of the AuNPs was measured at $400 \mathrm{~nm}$. In the third run (\#3), the dispersion of the AuNPs was measured at $520 \mathrm{~nm}$, in the fourth run (\#4), the $\mathrm{Au} / \mathrm{Ag}$ co-dispersion was measured at $400 \mathrm{~nm}$, and finally in the fifth run (\#5), the Au/Ag co-dispersion was measured at $520 \mathrm{~nm}$. Given that i) the AgNPs absorb strongly around $400 \mathrm{~nm}$ but weakly beyond $500 \mathrm{~nm}$, and ii) the AuNPs absorb strongly around $520 \mathrm{~nm}$, the apparent particle size (here: optical-absorption-weighted average) of the Au/Ag co-dispersion must be different when measured at these two wavelengths. Accordingly, at $400 \mathrm{~nm}$, the presence of AgNPs must be evident, while at $520 \mathrm{~nm}$, the presence of AuNPs must be dominant. Measuring the co-dispersion at different wavelengths therefore resulted in different apparent particle sizes (absorption-weighted average), proving that the particles were truly co-dispersed in the gradient profile.

\section{Experimental Section}

Chemicals: Gold (III) chloride trihydrate $\left(\mathrm{HAuCl}_{4} \cdot 3 \mathrm{H}_{2} \mathrm{O}, \geq 99.9 \%\right)$, sodium citrate tribasic dihydrate $\left(\mathrm{C}_{6} \mathrm{H}_{5} \mathrm{Na}_{3} \mathrm{O}_{7} \cdot 2 \mathrm{H}_{2} \mathrm{O}, \geq 98 \%\right)$, and hydroxylamine hydrochloride $\left(\mathrm{NH}_{2} \mathrm{OH} \cdot \mathrm{HCl}, \mathrm{ACS}\right.$ reagent, $\left.98 \%\right)$, silver nitrate $\left(\mathrm{AgNO}_{3}\right.$, ACS reagent, $\left.\geq 99 \%\right)$, tannic acid $\left(\mathrm{C7}_{6} \mathrm{H}_{52} \mathrm{O}_{46}, \mathrm{ACS}\right.$ reagent) and polyvinylpyrrolidone (PVP, Mw $=10000)$ were purchased from Sigma-Aldrich. Thiol-PEG-carboxylate and methoxy poly(ethylene glycol) $(\mathrm{Mw}=5000)$ were purchased from Creative PEGWorks. Milli-Q water was used in all preparations.

Synthesis of the AuNPs: The larger particles were prepared by seeded-growth method based on the procedure described in detail elsewhere. ${ }^{[41,42]}$ Briefly, $1.34 \mathrm{~mL}$ of hydroxylamine hydrochloride $(0.22 \mathrm{M})$ were added to $144 \mathrm{~mL}$ aqueous solution containing gold salt $\left(0.25 \times 10^{-3} \mathrm{M}\right), 15 \mathrm{~nm}$ gold seeds $\left([\mathrm{Au}]=0.0125 \times 10^{-3} \mathrm{M}\right)$ and sodium citrate $\left(0.5 \times 10^{-3} \mathrm{M}\right)$ under vigorous magnetic stirring. After $3 \mathrm{~h}$, a solution of SH-PEG-COOH sonicated previously for $30 \mathrm{~min}$ was added dropwise to the mixture and stirred overnight to coat the particles, resulting in a surface density of $\approx 25$ molecules $\mathrm{nm}^{-2}$. The dispersion was cleaned twice by centrifugation at $2800 \mathrm{rpm}$ for $35 \mathrm{~min}$ and redispersed in Milli-Q water. The small seeds (S-AuNPs) were prepared by the wellknown Turkevich method, ${ }^{[43]}$ where an aqueous solution of sodium citrate $\left(1.7 \times 10^{-3} \mathrm{M}\right)$ was added quickly to a boiling solution of gold salt $\left(0.5 \times 10^{-3} \mathrm{M}\right)$ under vigorous magnetic stirring. After $15 \mathrm{~min}$, the dispersion was allowed to cool down to room temperature. Particles were coated with 5 molecules $\mathrm{nm}^{-2} \mathrm{mPEC}$ following the same protocol used for the L-AuNPs. The excess of mPEG was removed by centrifugation at $10000 \mathrm{rpm}$ for $30 \mathrm{~min}$.

Synthesis of the AgNPs: The particles were synthetized by following the method described in detail elsewhere. ${ }^{[44]}$ Briefly, silver nitrate 
$\left(1.06 \times 10^{-3} \mathrm{M}\right)$ was refluxed at $130^{\circ} \mathrm{C}$ in the presence of tannic acid $\left(0.29 \times 10^{-3} \mathrm{M}\right)$ and sodium citrate $\left(2.45 \times 10^{-3} \mathrm{M}\right)$ for $20 \mathrm{~min}$. The NPs were coated with PVP by adding dropwise a solution of PVP to the dispersion (resulting in a surface density of approx. 18 molecules $\mathrm{nm}^{-2}$ ). After $4 \mathrm{~h}$, the dispersion was cleaned by centrifugation at $3500 \mathrm{rpm}$ for $25 \mathrm{~min}$, and redispersed in Milli-Q water.

UV-Vis Spectra: Spectra were recorded at $25^{\circ} \mathrm{C}$ in a Jasco V-670 spectrophotometer using $10 \mathrm{~mm}$ path length quartz Suprasil-grade cuvettes (Hellma Analytics).

Transmission Electron Microscopy: NPs were imaged by using a Tecnai Spirit transmission electron microscope (FEI, Hillsboro, OR, US) operating at $120 \mathrm{kV}$ equipped with a CCD camera (Eagle, ThermoFischer, Waltham, MA, US). Samples were prepared by drop casting $10 \mu \mathrm{L}$ of the dispersion onto a 300-mesh carbon-membrane-coated copper grid. The size distribution of the particles was estimated by using an open source image processing program (Image) $).[45,46]$

Observing Gradient Profiles in a Capillary-Electrophoresis System: Optical absorbance was recorded in a capillary electrophoresis system (Prince560CE Autosampler, Prince Technologies, B.V., Netherland), using a $145 \mathrm{~cm}$ long fused silica capillary with an internal diameter of $74.5 \mu \mathrm{m}$ (Polymicro Technologies, Phoenix, USA). The detection window (output) was $72 \mathrm{~cm}$ apart from the injection point (input), and an ActiPix D100 area detector (Paraytec, York, UK) in combination with a bandpass filter (either $520 \pm 10 \mathrm{~nm}$ or $400 \pm 10 \mathrm{~nm}$ ) and a neutral density filter of $10 \%$ transmission (Edmund Optics, York, UK) was used to record the optical absorbance.

In the first experiment (Figure 3), alternating sequences of AuNPs and Milli-Q water were injected into the capillary as input. AuNPs ([Au] $=0.6 \times 10^{-3} \mathrm{M} ; 200 \mathrm{mbar}$ for $0.08 \mathrm{~min}$ or $10 \mathrm{~min}$ ) and Milli-Q water (90 mbar for $8 \mathrm{~min}$ ). In the second experiment (Figure 4), AuNPs and AgNPs as well as their co-dispersion ( $\left.[\mathrm{Au}]=[\mathrm{Ag}]=0.6 \times 10^{-3} \mathrm{M}\right)$ were injected at $200 \mathrm{mbar}$ for $0.2 \mathrm{~min}$ and were driven through the capillary at 90 mbar for $20 \mathrm{~min}$. Milli-Q water was used as eluent for all the experiments. After each set of experiments, the fused silica capillary was cleaned by pumping $\mathrm{NaOH}(0.1 \mathrm{~m})$ at 2500 mbar for 10 min and then rinsed with Milli-Q water at $2500 \mathrm{mbar}$ for $10 \mathrm{~min}$.

Taylor Dispersion Analysis: To characterize the apparent hydrodynamic radius, the same capillary-electrophoresis system was used as described above, using two detection windows 37 and $72 \mathrm{~cm}$ apart from the injection point). Using two detection points promotes accuracy, ${ }^{[47,48]}$ and the apparent translation self-diffusion coefficient $D$ is estimated through the differences in variances and residence times of the gradient profiles recorded at the two windows: $D=Y^{2} / 24\left(t_{2}-t_{1}\right)\left(\sigma_{2}^{2}-\sigma_{1}^{2}\right)^{-1}$. The absorption-weighted average hydrodynamic radius ${ }^{[2,25]}$ is determined from $D$ via the Stokes-Einstein equation $\left(D=k_{\mathrm{B}} \cdot T / 6 \cdot \pi \cdot \eta \cdot r\right)$ where $\eta$ is the viscosity of the carrier fluid, $T$ is the temperature, and $k_{B}$ is the Boltzmann constant. ${ }^{[49-51]}$ Given that the optical extinction of a nanoparticle is a power function of the radius, $\mu(r, \lambda) \propto r^{n},{ }^{[52]}$ it can be shown that the absorption-weighted average hydrodynamic radius of polydisperse NPs scales as $\left\langle r^{4}\right\rangle /\left\langle r^{3}\right\rangle$, where $\left\langle r^{n}\right\rangle$ is the $\mathrm{n}^{\text {th }}$ raw moment of the particle size distribution $\left\langle r^{n}\right\rangle \equiv \int_{0}^{\infty} P(r) \cdot r^{n} \mathrm{~d} r$.

Data Availability: All data-raw and derived - can be obtained free of charge from the corresponding author.

\section{Acknowledgements}

The authors are grateful for the financial support of the Adolphe Merkle Foundation and the University of Fribourg. This work also benefitted from support of the Swiss National Science Foundation through the National Center of Competence in Research Bio-Inspired Materials.

\section{Conflict of Interest}

The authors declare no conflict of interest.

\section{Author Contributions}

S.B. conceived the project, developed the analytical approach, derived the theoretical treatment, and co-designed the Taylor dispersion experiments. P.T.B. synthesized the particles, analyzed TEM micrographs, carried out the UV-Vis experiments, co-designed and carried out the Taylor dispersion experiments. B.R.-R. and A.P.-F. supervised P.T.B. S.B. wrote the manuscript through contribution from the coauthors.

\section{Keywords}

bioinspiration, functional gradient materials, nanoparticles, soft nanocomposites, Taylor dispersion

[1] G. Udupa, S. S. Rao, K. V. Gangadharan, Procedia Mater. Sci. 2014, 5, 1291.

[2] A. Gupta, M. Talha, Prog. Aerospace Sci. 2015, 79, 1.

[3] M. Naebe, K. Shirvanimoghaddam, Appl. Mater. Today 2016, 5, 223.

[4] Z. Liu, M. A. Meyers, Z. Zhang, R. O. Ritchie, Prog. Mater. Sci. 2017, $88,467$.

[5] R. R. Naik, S. Singamaneni, Chem. Rev. 2017, 117, 12581

[6] M. A. da Silva, C. A. Dreiss, Polym. Int. 2016, 65, 268.

[7] S. Bhattacharya, S. K. Samanta, Chem. Rev. 2016, 116, 11967.

[8] A. C. Balazs, T. Emrick, T. P. Russell, Science 2006, 314, 1107.

[9] S. K. Kumar, B. C. Benicewicz, R. A. Vaia, K. I. Winey, Macromolecules 2017, 50, 714 .

[10] K. U. Claussen, T. Scheibel, H.-W. Schmidt, R. Giesa, Macromol. Mater. Eng. 2012, 297, 938.

[11] A. Spinnrock, M. Martens, F. Enders, K. Boldt, H. Cölfen, Nanomaterials 2019, 9, E988.

[12] C. Li, L. Ouyang, I. J. Pence, A. C. Moore, Y. Lin, C. W. Winter, J. P. K. Armstrong, M. M. Stevens, Adv. Mater. 2019, 31, 1900291

[13] A. Spinnrock, D. Schupp, H. Cölfen, Small 2018, 14, 1803518.

[14] G. Taylor, Proc. R. Soc. London, Ser. A 1953, 219, 186.

[15] G. Taylor, Proc. R. Soc. London, Ser. A 1954, 225, 473.

[16] R. Aris, Proc. R. Soc. London, Ser. A 1956, 235, 67.

[17] A. Ajdari, N. Bontoux, H. A. Stone, Anal. Chem. 2006, 78, 387.

[18] B. M. Belongia, J. C. Baygents, J. Colloid Interface Sci. 1997, 195, 19.

[19] F. d'Orlyé, A. Varenne, P. Gareil, J. Chromatogr. A 2008, 1204, 226.

[20] U. Pyell, A. H. Jalil, C. Pfeiffer, B. Pelaz, W. J. Parak, J. Colloid Interface Sci. 2015, 450, 288.

[21] U. Pyell, A. H. Jalil, D. A. Urban, C. Pfeiffer, B. Pelaz, W. J. Parak, J. Colloid Interface Sci. 2015, 457, 131.

[22] S. Balog, D. A. Urban, A. M. Milosevic, F. Crippa, B. Rothen-Rutishauser, A. Petri-Fink, J. Nanopart. Res. 2017, 19, 287.

[23] A. Fichtner, A. Jalil, U. Pyell, Langmuir 2017, 33, 2325.

[24] S. Balog, Anal. Chem. 2018, 90, 4258.

[25] D. A. Urban, A. M. Milosevic, D. Bossert, F. Crippa, T. L. Moore, C. Geers, S. Balog, B. Rothen-Rutishauser, A. Petri-Fink, Colloid Interface Sci. Commun. 2018, 22, 29.

[26] F. Oukacine, A. Gèze, L. Choisnard, J.-L. Putaux, J.-P. Stahl, E. Peyrin, Anal. Chem. 2018, 90, 2493.

[27] X. Zhang, X. Gao, L. Jiang, J. Qin, Langmuir 2012, 28, 10026.

[28] J. Goulpeau, B. Lonetti, D. Trouchet, A. Ajdari, P. Tabeling, Lab Chip 2007, 7, 1154

[29] Y. Du, M. J. Hancock, J. He, J. L. Villa-Uribe, B. Wang, D. M. Cropek, A. Khademhosseini, Biomaterials 2010, 31, 2686.

[30] C. Laval, A. Bouchaudy, J.-B. Salmon, Lab Chip 2016, 16, 1234. 
[31] P. Lemal, A. Petri-Fink, S. Balog, Anal. Chem. 2018, 91, 1217.

[32] P. Taladriz-Blanco, B. Rothen-Rutishauser, A. Petri-Fink, S. Balog, Anal. Chem. 2019, 91, 9946.

[33] K. L. Kelly, E. Coronado, L. L. Zhao, G. C. Schatz, J. Phys. Chem. B 2003, 107, 668.

[34] M. E. Mackay, A. Tuteja, P. M. Duxbury, C. J. Hawker, B. Van Horn, Z. Guan, G. Chen, R. S. Krishnan, Science 2006, 311, 1740.

[35] N. J. Fernandes, H. Koerner, E. P. Giannelis, R. A. Vaia, MRS Commun. 2013, 3, 13.

[36] C. Heinzmann, C. Weder, L. M. de Espinosa, Chem. Soc. Rev. 2016, 45, 342.

[37] R. Mezzenga, J. Ruokolainen, Nat. Mater. 2009, 8, 926.

[38] Y. Zhao, K. Thorkelsson, A. J. Mastroianni, T. Schilling, J. M. Luther, B. J. Rancatore, K. Matsunaga, H. Jinnai, Y. Wu, D. Poulsen, J. M. J. Fréchet, A. Paul Alivisatos, T. Xu, Nat. Mater. 2009, 8, 979.

[39] X. Wang, Z. Liu, Y. Pang, RSC Adv. 2017, 7, 29966.

[40] J. Garrison, Z. Li, B. Palanisamy, L. Wang, E. Seker, J. Biol. Eng. 2018, 12, 31.
[41] K. R. Brown, M. J. Natan, Langmuir 1998, 14, 726.

[42] K. R. Brown, L. A. Lyon, A. P. Fox, B. D. Reiss, M. J. Natan, Chem. Mater. 2000, 12, 314.

[43] B. V. Enustun, J. Turkevich, J. Am. Chem. Soc. 1963, 85, 3317.

[44] L. Rainville, M.-C. Dorais, D. Boudreau, RSC Adv. 2013, 3, 13953.

[45] J. Schindelin, C. T. Rueden, M. C. Hiner, K. W. Eliceiri, Mol. Reprod. Dev. 2015, 82, 518

[46] C. A. Schneider, W. S. Rasband, K. W. Eliceiri, Nat. Methods 2012 9, 671 .

[47] U. Sharma, N. J. Gleason, J. D. Carbeck, Anal. Chem. 2005, 77, 806.

[48] J. Chamieh, H. Cottet, J. Chromatogr. A 2012, 1241, 123.

[49] A. Einstein, Ann. Phys. 1905, 17, 549.

[50] A. Einstein, Ann. Phys. 1906, 324, 289.

[51] A. Einstein, Ann. Phys. 1911, 339, 591.

[52] M. Quinten, Optical Properties of Nanoparticle Systems: Mie and Beyond, Wiley-VCH Verlag GmbH, Weinheim 2011. 